\title{
Application of indocyanine green injection guided by electromagnetic navigation bronchoscopy in localization of pulmonary nodules
}

\author{
Jie Zhang ${ }^{1,2,3 \#}$, Jiaxi He ${ }^{1,2,3 \#}$, Jianfeng Chen ${ }^{2,3,4 \#}$, Yunpeng Zhong ${ }^{1,2,3}$, Jianxing $\mathrm{He}^{1,2,3}$, Shuben $\mathbf{L i}^{1,2,3}$ \\ ${ }^{1}$ Department of Thoracic Surgery, the First Affiliated Hospital of Guangzhou Medical University, Guangzhou, China; ${ }^{2}$ Guangzhou Institute of \\ Respiratory Disease \& China State Key Laboratory of Respiratory Disease, Guangzhou, China; ${ }^{3}$ National Clinical Research Center for Respiratory \\ Disease, Guangzhou, China; ${ }^{4}$ Department of Anesthesiology, the First Affiliated Hospital of Guangzhou Medical University, Guangzhou, China \\ Contributions: (I) Conception and design: J He, S Li; (II) Administrative support: S Li, J He; (III) Provision of study materials or patients: J Zhang, \\ J He; (IV) Collection and assembly of data: J Zhang, J He, J Chen; (V) Data analysis and interpretation: J Zhang, J He, J Chen; (VI) Manuscript \\ writing: All authors; (VII) Final approval of manuscript: All authors. \\ \#These authors contributed equally to this work. \\ Correspondence to: Shuben Li, MD, PhD; Jianxing He, MD, PhD. Department of Thoracic Surgery, the First Affiliated Hospital of Guangzhou \\ Medical University, Guangzhou, China. Email: 13500030280@163.com; hejianxing@hotmail.com.
}

Background: Accurate localization of pulmonary nodules becomes a crucial step in diagnosis and
treatment. However, the efficacy and accuracy of electromagnetic navigation bronchoscopy (ENB)-guided
localization with indocyanine green (ICG) injection for video-assisted thoracoscopic surgery (VATS) lung
resection remain unclear.
Methods: A retrospective study was performed that patients who had small pulmonary nodules and
underwent lung resection after ENB-guided localization were included from Oct 2018 to Mar 2021 . The
analysis of the efficacy and accuracy of ENB-guided localization was conducted.
Results: A total of 181 pulmonary nodules in 173 patients were recorded which were $9.21 \pm 4.81$ mm in size.
The mean time of ENB-guided localization was $7.99 \pm 4.9$ minutes. The success rate of nodule localization
was $98.3 \%$ (178/181), while the accuracy was $89 \%(161 / 181$ ) without any complication. All patients received
thoracoscopic surgery after localization, and all nodules were completely resected. A customized scoring
system was used to evaluate localization accuracy, and the patients were divided into four groups according to
it. The localization accuracy was positively associated with the bronchus sign $(\mathrm{P}<0.001$ ) and negatively with
the location of the nodule (anterior segment and superior lingual segment of left upper lobe) (P=0.013 and
0.03 , respectively).

Conclusions: ENB-guided pulmonary nodule localization by ICG injection is an accurate and effective method with a short operation time and few complications, which could be widely used in clinical practice.

Keywords: Electromagnetic navigation bronchoscopy; pulmonary nodules localization; video-assisted thoracoscopic surgery

Submitted Aug 31, 2021. Accepted for publication Nov 12, 2021.

doi: $10.21037 /$ tlcr-21-699

View this article at: https://dx.doi.org/10.21037/tlcr-21-699

\section{Introduction}

Surgical resection of pulmonary nodules is beneficial to operable early-stage lung cancer patients (1). However, some nodules are small and difficult to identify via intraoperative finger palpation or instrument sliding (2). Therefore, preoperative localization of them is a crucial step for the sake of precise and minimal invasiveness. Existing techniques for localization of pulmonary nodules 
mainly include: computer tomography (CT)-guided localization, intraoperative ultrasound-assisted localization, and navigational bronchoscopy-guided localization (3-5), each has its benefits and drawbacks.

CT-guided preoperative localization is widely performed in clinical practice because of its technical maturity, simple operating procedure, short time-cost, and high success rate. However, its application has limitations and risks, including severe pain, hemorrhage, hemopneumothorax, needle track tumor implantation (6), and markers displacement (7). Besides, some localizations are challenging due to anatomical issues like those next to the great vessels or beneath the scapula and sternum. As for ultrasoundassisted localization, the resolution of ultrasound would be significantly affected by the air retention in those patients with pulmonary bullae, diffuse emphysema, and pulmonary fibrosis $(5,8)$. Therefore, ultrasound-assisted localization is now rarely used before pulmonary nodule resections.

Contemporarily, the electromagnetic navigation bronchoscopy (ENB) guided pulmonary nodule localization has been proved to be a safe, effective, and minimally invasive technique (9-11). Indocyanine green (ICG), a non-toxic near-infrared fluorescent dye, has already been applied in the localization of pulmonary nodules and reported to be stable and better than other dyes in the aspect of the pathological exam $(4,12)$. However, the efficacy and accuracy of ENGguided ICG injection for pulmonary nodule localization remain unclear. We, therefore, performed a study to evaluate the effectiveness and accuracy of ENB-guided ICG injection for the localization of pulmonary nodules.

We present the following article in accordance with the STROBE reporting checklist (available at https://dx.doi. org/10.21037/tlcr-21-699).

\section{Methods}

\section{Patient selection}

A single-center retrospective study was conducted that the patients who had received preoperative ENB-guided localization in our hospital from Oct 2018 to Mar 2021were included. The inclusion criteria were: (I) patients aged older than 18; (II) chest CT indicated the lung lesions were less than $2 \mathrm{~cm}$ in diameter and more than $10 \mathrm{~mm}$ from the visceral pleura; (III) preoperative chest CT revealed that the lesions were challenging to localize or identify with observation or palpation during surgery. For example: the surgeon could not reach the nodule with the finger; nodules away from the visceral pleura that are difficult to observe or touch; the nodules were mainly ground glass opacity, which were difficult to identify by touch; (IV) no operational contradiction was reported. Exclusion criteria included: (I) patients in poor general condition, unable to tolerate bronchoscopy; (II) regional invasion or distant metastasis was observed in preoperative assessment.

The study was conducted following the Declaration of Helsinki (as revised in 2013). The study was approved by the Ethics Committee of the Guangzhou Medical University First Affiliated Hospital (No. 2020K-43), and individual consent for this retrospective analysis was waived.

\section{ENB localization procedure}

(I) Preoperative navigation track planning: preoperative chest CT (Definition AS+128, Siemens, Germany; or Revolution 256, GE, USA) scan was performed, the DICOM images were extracted and simulated in the ENB system (LUNGCARE, Suzhou, China) before the operation. The three-dimensional coordinates and a virtual bronchial tree with target lesions were established, and a navigation track was determined through the appropriate bronchus and bronchioles. The distance from the end of the bronchus to the lesion would be recorded and demonstrated.

(II) Registration: the patient was placed supine decubitus on the examining bed and underwent the fiberoptic bronchoscopy under spontaneous respiratory anesthesia with laryngeal mask. The fiberoptic bronchoscope was placed above the carina, the magnetic guiding wire and sheath were inserted through the bronchoscopic working channel. The procedure would be completed till the bilateral main bronchus and trachea were registered subsequently via the guiding wire.

(III) Localization: operate the bronchoscopy to the tertiary bronchus or distal bronchiole and insert the guiding wire with the sheath to the target lesion according to the navigation guidance. After reaching the target area, the guiding wire was withdrawn with the sheath fixed in the working channel. A total of $0.3 \mathrm{~mL}$ indocyanine green (0.6 mg/mL; Dandong Yichuang Pharmaceutical Co., Ltd., China) was injected following $2 \mathrm{ml}$ air through the guide sheath. The guiding wire was reapplied to the sheath to reconfirm the staining area, and the localization was completed. The distance from 
Table 1 Localization accuracy scoring system, ICG, indocyanine green

\begin{tabular}{lcc}
\hline Accuracy score & ICG tracing & The distance from the marker to the lesion \\
\hline 1 & No tracer & - \\
2 & Tracer shown & Distance $>25 \mathrm{~mm}$ \\
3 & Tracer shown & $15 \mathrm{~mm}<$ distance $\leq 25 \mathrm{~mm}$ \\
4 & Tracer shown & $5 \mathrm{~mm}<$ distance $\leq 15 \mathrm{~mm}$ \\
5 & Tracer shown & distance $\leq 5 \mathrm{~mm}$ \\
\hline
\end{tabular}

the location to the lesion were recorded, and the localization accuracy scoring system was shown in Table 1. Accuracy scores of 4 and 5 were identified as accurate. Successful localization indicates the following: the airway condition shown by the navigation system matches the actual situation, and we can locate through the optimal route designed by the navigation system.

\section{Surgical procedure}

The localization and surgical procedure were operated in the same operating room (OR). After localization, the patients were transferred to the operating table, and received video-assisted thoracoscopic surgery (VATS) sublobar resections. An infrared fluorescent thoracoscope (Stryker, Kalamazoo, MI, USA) was used to detect the ICG marker. The intraoperative frozen section results determined the resection area.

\section{Statistical analysis}

Clinical data were reported as count (percentage) or mean \pm standard deviation values. The patients were divided into four groups according to the localization accuracy score. Measurement data were compared using the one-way analysis of variance. The Chi-square test or Fisher exact test was applied for categorical data. Multivariate analysis was performed using ordinal logistic regression analysis. $\mathrm{P}$ values less than 0.05 were considered statistically significant. IBM SPSS version 25 software (IBM Corporation, Somers, NY, USA) was used for statistical analyses.

\section{Results}

\section{Basic characteristics of patients}

A total of 181 nodules from 173 patients (110 females, 63 males) (Table 2) were included in the study. The distribution of nodules in each lung segment was shown in Figure 1. The mean size of the nodules was $9.21 \pm 4.81 \mathrm{~mm}$. A large proportion of nodules were pure ground-glass opacity (pGGO) and mixed GGO (mGGO) in the CT images, which were $92(50.8 \%)$ and 83 (45.9\%), respectively. Only 6 cases $(3.3 \%)$ were solid nodules. Most (170, 93.9\%) nodules were adjacent to the bronchus. The average distance between the bronchus and the nodules was $24.91 \pm 16.49 \mathrm{~mm}$. But only 11 nodules showed a bronchial sign indicating a bronchus passing through or reaching the nodule.

\section{Operation characteristics of patients}

All localizations were completed successfully with a short registration time $(0.97 \pm 0.43 \mathrm{~min})$. The localization time was shown in Figure 2. Most $(157,90.8 \%)$ patients had a single target, ten patients had two targets, and six patients had three more targets. The average localization time became longer as the number of targets increased (Table 3), $9.75 \pm 6.5,11.35 \pm 5.27,24.54 \pm 17.05 \mathrm{~min}$, respectively. The average distance from the marker to the targets was $33.8 \pm 10.57 \mathrm{~mm}$. The majority of nodules $(178 / 181$, $98.3 \%$ ) were successfully localized, and 161 were identified as accurate. No hemorrhage, pneumothorax, and other complications occurred in any cases. The patients underwent VATS after localization, and all nodules were completely resected.

\section{Comparison of parameters among four groups with different accuracy scores}

The clinical characteristics of the four groups were summarized in Table 4. The four groups did not significantly differ in age, gender, nodule size, nodule location, nodule nature, and other general conditions $(\mathrm{P}>0.05)$. The patients with bronchial signs had significantly higher accuracy scores 
Table 2 Basic characteristics of patients

\begin{tabular}{|c|c|}
\hline Characteristic & Value \\
\hline Total number (patients/nodules) & $173 / 181$ \\
\hline Age, (years) & $52.77 \pm 11.08$ \\
\hline \multicolumn{2}{|l|}{ Gender, n (\%) } \\
\hline Male & $63(36.4)$ \\
\hline Female & $110(63.4)$ \\
\hline Nodule size (mm) & $9.21 \pm 4.81$ \\
\hline \multicolumn{2}{|l|}{ Characteristics, n (\%) } \\
\hline pGGN & $92(50.8)$ \\
\hline mGGN & $83(45.9)$ \\
\hline Solid nodule & $6(3.3)$ \\
\hline \multicolumn{2}{|l|}{ Nodules location, n (\%) } \\
\hline RUL & $63(34.8)$ \\
\hline RML & $11(6.1)$ \\
\hline RLL & $35(19.3)$ \\
\hline LUL & $39(21.5)$ \\
\hline LLL & $33(18.2)$ \\
\hline \multicolumn{2}{|l|}{ Bronchus sign, n (\%) } \\
\hline Yes & $11(6.1)$ \\
\hline No & $170(93.9)$ \\
\hline Distance from bronchus to nodule (mm) & $24.91 \pm 16.49$ \\
\hline \multicolumn{2}{|l|}{ Pathology, n (\%) } \\
\hline $\mathrm{AAH}$ & $10(5.5)$ \\
\hline AIS & $17(9.4)$ \\
\hline MIA & $82(45.3)$ \\
\hline IA & $34(18.8)$ \\
\hline Benign & $35(19.3)$ \\
\hline Metastatic carcinoma & $3(1.7)$ \\
\hline
\end{tabular}

pGGN, pure ground-grass nodule; mGGN, mixed groundgrass nodule; RUL, right upper lobe; RML, right middle lobe; RLL, right lower lobe; LUL, left upper lobe; LLL, left lower lobe; $\mathrm{AAH}$, atypical adenomatous hyperplasia; AIS, adenocarcinoma in situ; MIA, minimally invasive adenocarcinoma; IA, invasive adenocarcinoma.

$(\mathrm{P}<0.01)$. The results of pairwise comparisons showed that the distance from the bronchus to the lesion was greater in the group scoring 5 than in the group scoring $4(\mathrm{P}<0.05)$, more nodules in group scoring 5 had bronchial signs compared with nodules with accuracy scores of 3 and $4(\mathrm{P}<0.05$ and $\mathrm{P}<0.01$ ), and the localization success rate was significantly lower in the group with a score of 2 than in the groups with precise localization $(\mathrm{P}<0.01$ and $\mathrm{P}<0.05)$ (Table $\mathrm{S} 1)$.

\section{Results of ordinal logistic regression analysis}

On the premise of a good fitting effect (Pearson test, $\mathrm{P}=1$ ) of the regression model, the ordinal logistic regression analysis results were shown in Table S2. Nodules with bronchial signs had higher localization accuracy scores (estimate $=3.283, \mathrm{P}<0.001$ ). In contrast, the localization accuracy was lower when the nodules were located in the left anterior segment (estimate $=-2.708, \mathrm{P}=0.013$ ) and the superior lingular segment (estimate $=-2.5, \mathrm{P}=0.03$ ). SPSS software was used to obtain the distribution table of the actual score and predicted score frequency of these data. The accuracy of nodule localization determined by three significant factors was 79\% (Table 5).

\section{Discussion}

The practice rationale of ENB-guided localization is the electromagnetic technology and high-resolution chest CT scan. A virtual tracheobronchial tree would be reconstructed from CT images, and the path to the target lesions is created to guide the bronchoscopy. Compared with CT-guided localization, the ENB is performed under general anesthesia, which can alleviate the psychological pressure and pain of the patients. The localization and surgical procedure are operated in the operating room (OR) by the same team in our center, which simplifies the treatment process without unnecessary transportation from the CT scan room to OR (11). Besides, ENB also eliminates radiation exposure and markedly decreases puncturerelated complications since it is operated via natural orifice (13). ENB localization cost $7.99 \pm 4.9 \mathrm{~min}$ on average in the current study, and the operation time was shorter than the CT-guided method, which was about 18 minutes in some reports (4). In the previous study, the incidence of complications of CT-guided localization, including pulmonary hemorrhage, pneumothorax, hemoptysis, and pleural reaction, was $6.8 \%, 5.9 \%, 0.4 \%$, $0.4 \%$, respectively (4). In contrast, no complication was reported in this study.

Comparing to conventional bronchoscopy, ENB can enter the quaternary bronchioles or beyond under the guidance of a magnetic system, which makes it more precise 
A

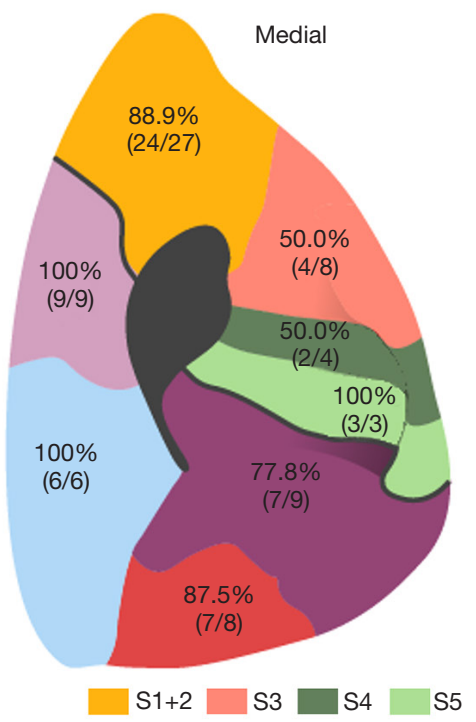

B

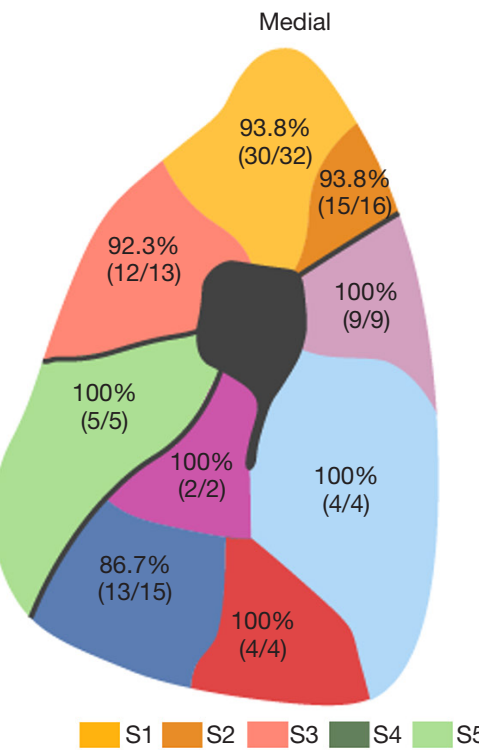

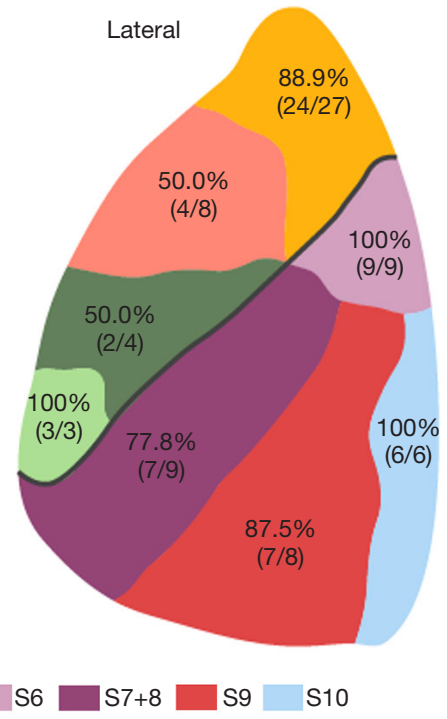

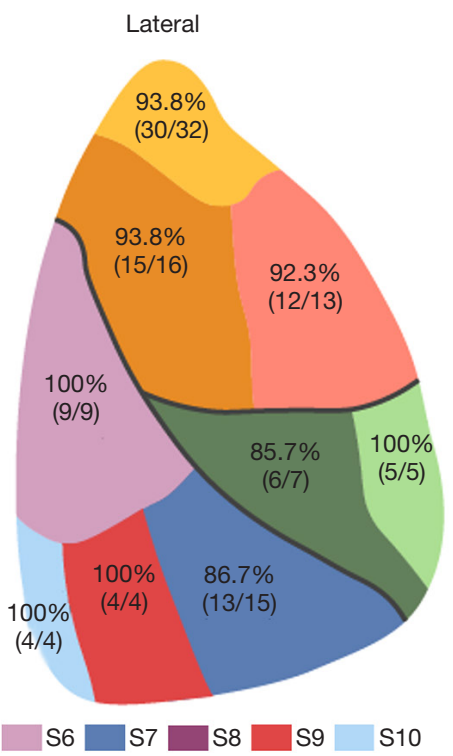

Figure 1 The distribution and localization accuracy of nodules in each lung segment. (A) Medial and lateral aspects of the left lung segments; (B) medial and lateral aspects of the right lung segments.

and accurate (14-16). A success rate of $97.2 \%$ was reported in a study by Marino et al., which included 72 nodules with diameters less than $20 \mathrm{~mm}$ (17). Awais et al. used methylene blue to localize the pulmonary nodules via ENB before the VATS small pulmonary nodule resection. The pulmonary nodules of 29 patients were successfully located and completely resected (18). In our study, a total of 178 out of 181 nodules were successfully located in 173 patients. The success rate was $98.3 \%$, which was similar to the CTguided localization (4). There were 162 nodules with accuracy scores of 4 and 5, which accounted for $89 \%$ of the total cohort. But it should be noticed that the distance between localization and lesion was longer in the inflation phase during the localization process. Such distance would be measured again after resection when the lung tissue was completely deflated. Thus, the actual proportion of scoring 4 or 5 in our cohort should be higher than $89 \%$. Three cases were recorded as failed, including 1 case of congenital bronchial variation defect and 1 case of occluded periphery bronchial branch that the target had no way to reach. 


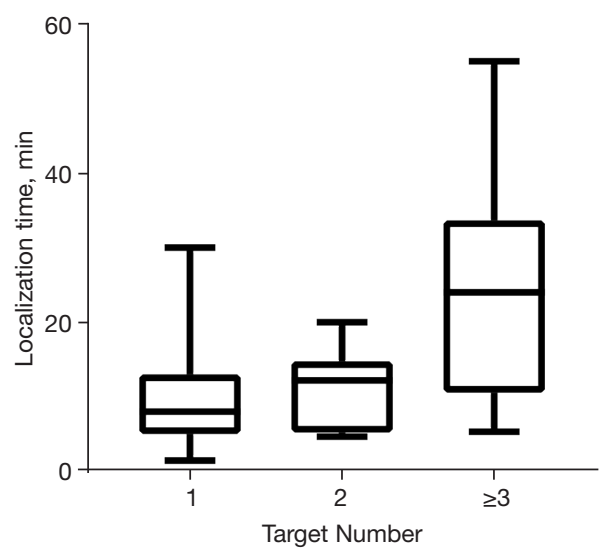

Figure 2 The localization time of different target number.

Table 3 Operation characteristics of patients

\begin{tabular}{lc}
\hline Characteristic & Value \\
\hline Registration time $(\mathrm{min})$ & $0.97 \pm 0.43$ \\
Localization time (min) & \\
1 nodule $(\mathrm{n}=157)$ & $9.75 \pm 6.5$ \\
2 nodules $(\mathrm{n}=10)$ & $11.35 \pm 5.27$ \\
$\geq 3$ nodules ( $\mathrm{n}=6)$ & $24.54 \pm 17.05$ \\
Distance from location to nodule (mm) & $33.8 \pm 10.57$ \\
Localization accuracy score, $\mathrm{n}(\%)$ & \\
2 & $5(2.8)$ \\
3 & $15(8.3)$ \\
4 & $134(74.0)$ \\
5 & $27(14.9)$ \\
Successful localization, $\mathrm{n}(\%)$ & $178(98.3)$ \\
Operation mode, $\mathrm{n}(\%)$ & \\
Wedge resection & $171(94.4)$ \\
Segmentectomy & $7(3.9)$ \\
Lobectomy & $3(1.7)$ \\
\hline
\end{tabular}

The last case was unsuccessful because the large-sized bronchoscopy could not enter the tertiary bronchiole with a small diameter and a steep angle.

In addition, we used methylene blue (MB) staining in some cases at the very beginning. We used a small dose of MB to achieve precise localization (19). However, some nodules are difficult to observe because of the long distance from the pleura and poor penetration of MB (20).
Besides, it is difficult for pathologists to identify lesions in the methylene blue-stained area while fluorescent dyes in their natural state will not trouble pathologists in dyeing and diagnosis. Infrared fluorescence also has a higher penetration to the tissue and can be easily recognized. Therefore, we now prefer to inject ICG for localization.

In a previous study, we found that the lesions that were more distant from the bronchus or located in the upper lobe were more difficult to localize (21). In this study, we tried to investigate the factors affecting localization accuracy with ordinal logistic regression analysis. Therefore, we analyzed nodule size, nodule location, bronchial sign, and distance between the lesions and bronchus. Due to conflict over the latter two variables, we built two analytical models separately. Unfortunately, only the model containing the bronchus sign was successfully established. The most accurate group had bronchial ends that were more distant from the lesion may account for this phenomenon. When there is no bronchus leading to the lesion, we select the closest point of the adjacent bronchus for localization. It turns out that this approach has been proved feasible, and $0.3 \mathrm{ml}$ of dye did not cause extensive dissemination. Owing to the distal bronchial distribution, the staining area might appear to be not satisfied though the distance from the bronchus to the lesion is relatively short. Of course, the insufficient number of cases can also lead to a mismatch, which needs to be analyzed with more cases.

Regression analysis results showed that the accuracy of nodule localization was improved when there was a bronchial sign. Nodules located in the anterior segment of the left upper lobe and the superior lingual segment were more challenging to localize accurately. Considering that the nodules at some sites require relatively high operating skills, a better clinical effect will be obtained if the locatable wire with optional angulation can be designed. At present, some systems have been available to enable the navigation probe to pass through some complex bending angles (22).

\section{Strengths and limitations}

To the best of our knowledge, this is the largest study to report on preoperative ENB-guided localization of pulmonary nodules by ICG injection for minimally invasive surgical resection. There are also some limitations of the ENB-guided localization technique. ENB-guided localization costs more than CT-guided localization, and the localization might be failed in case of the abnormal airway. Also, ENB requires an experienced and skillful operator. 
Table 4 Characteristics of groups with different localization accuracy score

\begin{tabular}{|c|c|c|c|c|c|}
\hline \multirow{2}{*}{ Characteristic } & \multicolumn{4}{|c|}{ Accuracy score } & \multirow{2}{*}{$\mathrm{P}$} \\
\hline & 2 points & 3 points & 4 points & 5 points & \\
\hline Gender, n (\%) & & & & & 0.227 \\
\hline Female & $3(60.0)$ & $8(53.3)$ & $90(67.2)$ & $13(48.1)$ & \\
\hline Nodule size (mm) & $9.2 \pm 4.6$ & $8.6 \pm 5.28$ & $9.24 \pm 4.84$ & $9.41 \pm 4.71$ & 0.962 \\
\hline Nodule nature, n (\%) & & & & & 0.249 \\
\hline pGGN & $2(40.0)$ & $6(40.0)$ & $67(50.0)$ & 17 (63.0) & \\
\hline Nodules location, n (\%) & & & & & 0.613 \\
\hline RUL & $1(20.0)$ & $5(33.3)$ & $47(35.1)$ & $10(37.0)$ & \\
\hline RML & $0(0)$ & $1(6.7)$ & $8(8.1)$ & $2(7.4)$ & \\
\hline RLL & $0(0)$ & $4(26.7)$ & $23(17.2)$ & $8(29.6)$ & \\
\hline LUL & $3(60.0)$ & $4(26.7)$ & 28 (20.9) & $4(14.8)$ & \\
\hline LLL & $1(20.0)$ & $1(6.7)$ & $28(20.9)$ & $3(11.1)$ & \\
\hline Pathology, n (\%) & & & & & 0.665 \\
\hline AAH & $0(0)$ & $2(13.3)$ & $7(5.2)$ & $1(3.7)$ & \\
\hline AIS & $1(20.0)$ & $1(6.7)$ & $14(10.4)$ & $1(3.7)$ & \\
\hline MIA & $1(20.0)$ & $5(33.3)$ & $61(45.5)$ & $15(55.6)$ & \\
\hline $\mathrm{IA}$ & $1(20.0)$ & $4(26.7)$ & $24(17.9)$ & $5(18.5)$ & \\
\hline Benign & $2(40.0)$ & $2(13.3)$ & $26(19.4)$ & $5(18.5)$ & \\
\hline Metastatic carcinoma & $0(0)$ & $1(6.7)$ & $2(1.5)$ & $0(0)$ & \\
\hline Successful localization & $3(60.0)$ & $14(93.3)$ & $134(100.0)$ & $27(100.0)$ & 0.000 \\
\hline
\end{tabular}

pGGN, pure ground-grass nodule; mGGN, mixed ground-grass nodule; RUL, right upper lobe; RML, right middle lobe; RLL, right lower lobe; LUL, left upper lobe; LLL, left lower lobe; AAH, atypical adenomatous hyperplasia; AIS, adenocarcinoma in situ; MIA, minimally invasive adenocarcinoma; IA, invasive adenocarcinoma. 
Table 5 Predicted response category crosstabulation

\begin{tabular}{lccc}
\hline \multirow{2}{*}{ Accuracy score } & \multicolumn{2}{c}{ Predicted response category } & Total \\
\cline { 2 - 3 } & 4 & 5 & \\
\hline 2 & 5 & 0 & 5 \\
3 & 15 & 0 & 15 \\
4 & 133 & 1 & 134 \\
5 & 17 & 10 & 27 \\
Total & 170 & 11 & 181 \\
\hline
\end{tabular}

The accuracy of localization and operation time is closely related to the surgeons. Therefore, systematic training and continuous practicing to accumulate experience are required. Encouragingly, our previous study showed that this technique would be mastered shortly, demonstrated by higher success and accuracy rates and shorter operation times (21). Lastly, the study was retrospective, which might have selection bias.

\section{Conclusions}

Our study showed that ENB-guided localization of pulmonary nodules by ICG injection is an accurate and effective method with a short operation time and few complications. Although there are limitations, it can provide an option for precise preoperative localization of lung resection surgery for small pulmonary nodules. Further investigation and clinical experience accumulation are warranted to understand better and improve such techniques.

\section{Acknowledgments}

Funding: This study was supported by National Key R\&D Program of China (2017YFC0112700).

\section{Footnote}

Reporting Checklist: The authors have completed the STROBE reporting checklist. Available at https://dx.doi. org/10.21037/tlcr-21-699

Data Sharing Statement: Available at https://dx.doi. org/10.21037/tlcr-21-699

Peer Review File: Available at https://dx.doi.org/10.21037/ tlcr-21-699

Conflicts of Interest: All authors have completed the ICMJE uniform disclosure form (available at https://dx.doi. org/10.21037/tlcr-21-699). The authors have no conflicts of interest to declare.

Ethical Statement: The authors are accountable for all aspects of the work in ensuring that questions related to the accuracy or integrity of any part of the work are appropriately investigated and resolved. The study was conducted in accordance with the Declaration of Helsinki (as revised in 2013). The study was approved by the Ethics Committee of the Guangzhou Medical University First Affiliated Hospital (No. 2020K-43), and individual consent for this retrospective analysis was waived.

Open Access Statement: This is an Open Access article distributed in accordance with the Creative Commons Attribution-NonCommercial-NoDerivs 4.0 International License (CC BY-NC-ND 4.0), which permits the noncommercial replication and distribution of the article with the strict proviso that no changes or edits are made and the original work is properly cited (including links to both the formal publication through the relevant DOI and the license). See: https://creativecommons.org/licenses/by-nc-nd/4.0/.

\section{References}

1. International Early Lung Cancer Action Program Investigators; Henschke CI, Yankelevitz DF, et al. Survival of patients with stage I lung cancer detected on CT screening. N Engl J Med 2006;355:1763-71.

2. Ciriaco P, Negri G, Puglisi A, et al. Video-assisted thoracoscopic surgery for pulmonary nodules: rationale for preoperative computed tomography-guided hookwire localization. Eur J Cardiothorac Surg 2004;25:429-33.

3. Hachey KJ, Digesu CS, Armstrong KW, et al. A novel technique for tumor localization and targeted lymphatic mapping in early-stage lung cancer. J Thorac Cardiovasc Surg 2017;154:1110-8.

4. Li X, Xu K, Cen R, et al. Preoperative computer tomography-guided indocyanine green injection is associated with successful localization of small pulmonary nodules. Transl Lung Cancer Res 2021;10:2229-36.

5. Liu C, Pu Q, Liu L. Is intracavitary thoracoscopic ultrasonography really needed for every intraparenchymal pulmonary nodule? J Thorac Cardiovasc Surg 
2013;145:1151.

6. Park CH, Han K, Hur J, et al. Comparative Effectiveness and Safety of Preoperative Lung Localization for Pulmonary Nodules: A Systematic Review and Metaanalysis. Chest 2017;151:316-28.

7. Wang L, Zhang X, Li M, et al. Remedial localization after dislodgement of primary mechanical localization in lung surgery. J Thorac Dis 2017;9:1240-6.

8. Wada H, Anayama T, Hirohashi K, et al. Thoracoscopic ultrasonography for localization of subcentimetre lung nodules. Eur J Cardiothorac Surg 2016;49:690-7.

9. Bolton WD, Howe H 3rd, Stephenson JE. The utility of electromagnetic navigational bronchoscopy as a localization tool for robotic resection of small pulmonary nodules. Ann Thorac Surg 2014;98:471-5; discussion 475-6.

10. Anayama T, Qiu J, Chan H, et al. Localization of pulmonary nodules using navigation bronchoscope and a near-infrared fluorescence thoracoscope. Ann Thorac Surg 2015;99:224-30.

11. Bolton WD, Cochran T, Ben-Or S, et al. Electromagnetic Navigational Bronchoscopy Reduces the Time Required for Localization and Resection of Lung Nodules. Innovations (Phila) 2017;12:333-7.

12. He J, Yang H, He J, et al. Electromagnetic navigation bronchoscopy fluorescence localization and VATS subxiphoid bilateral wedge resection under non-intubated anesthesia. J Thorac Dis 2019;11:3186-90.

13. Kuo SW, Tseng YF, Dai KY, et al. Electromagnetic Navigation Bronchoscopy Localization Versus Percutaneous CT-Guided Localization for Lung Resection via Video-Assisted Thoracoscopic Surgery: A PropensityMatched Study. J Clin Med 2019;8:379.

14. Mahajan AK, Patel S, Hogarth DK, et al. Electromagnetic navigational bronchoscopy: an effective and safe approach to diagnose peripheral lung lesions unreachable by conventional bronchoscopy in high-risk patients. J Bronchology Interv Pulmonol 2011;18:133-7.

15. Weiser TS, Hyman K, Yun J, et al. Electromagnetic navigational bronchoscopy: a surgeon's perspective. Ann Thorac Surg 2008;85:S797-801.

16. Reynisson PJ, Leira HO, Hernes TN, et al. Navigated bronchoscopy: a technical review. J Bronchology Interv Pulmonol 2014;21:242-64.

17. Marino KA, Sullivan JL, Weksler B. Electromagnetic Navigation Bronchoscopy for Identifying Lung Nodules for Thoracoscopic Resection. Ann Thorac Surg 2016;102:454-7.

18. Awais O, Reidy MR, Mehta K, et al. Electromagnetic Navigation Bronchoscopy-Guided Dye Marking for Thoracoscopic Resection of Pulmonary Nodules. Ann Thorac Surg 2016;102:223-9.

19. Krimsky WS, Minnich DJ, Cattaneo SM, et al. Thoracoscopic detection of occult indeterminate pulmonary nodules using bronchoscopic pleural dye marking. J Community Hosp Intern Med Perspect 2014.

20. Liu YH, Xu FP, Liao N, et al. Efficacy of intraoperative GeneSearch Breast Lymph Node (BLN) Assay for breast cancer metastasis detection in sentinel lymph node in Chinese patients. Cancer Sci 2010;101:1920-4.

21. Shi J, He J, He J, et al. Electromagnetic navigation-guided preoperative localization: the learning curve analysis. J Thorac Dis 2021;13:4339-48.

22. Chaddha U, Kovacs SP, Manley C, et al. Robot-assisted bronchoscopy for pulmonary lesion diagnosis: results from the initial multicenter experience. BMC Pulm Med 2019;19:243.
Cite this article as: Zhang J, He J, Chen J, Zhong Y, He J, Li S. Application of indocyanine green injection guided by electromagnetic navigation bronchoscopy in localization of pulmonary nodules. Transl Lung Cancer Res 2021;10(12):44144422. doi: 10.21037/tlcr-21-699 
Supplementary

Table S1 The pairwise comparisons of groups with different localization accuracy score

\begin{tabular}{|c|c|c|c|c|c|c|}
\hline Characteristic & \multicolumn{6}{|c|}{$\mathrm{P}$} \\
\hline Age (years) & 0.152 & 0.199 & 0.338 & 0.566 & 0.394 & 0.575 \\
\hline Gender, n (\%) & 1 & 1 & 1 & 0.284 & 0.747 & 0.06 \\
\hline Nodule size (mm) & 0.811 & 0.986 & 0.930 & 0.073 & 0.606 & 0.869 \\
\hline Nodules location, n (\%) & 0.441 & 0.311 & 0.170 & 0.679 & 0.912 & 0.486 \\
\hline Bronchus sign, n (\%) & N/A & 1 & 0.155 & 1 & 0.02 & 0.000 \\
\hline $\begin{array}{l}\text { Distance from } \\
\text { bronchus to nodule (mm) }\end{array}$ & 0.284 & 0.195 & 0.896 & 0.89 & 0.081 & 0.011 \\
\hline
\end{tabular}

$\mathrm{N} / \mathrm{A}$ : No statistics are computed because the variable is a constant. 
Table S2 The ordinal logistic regression analysis results

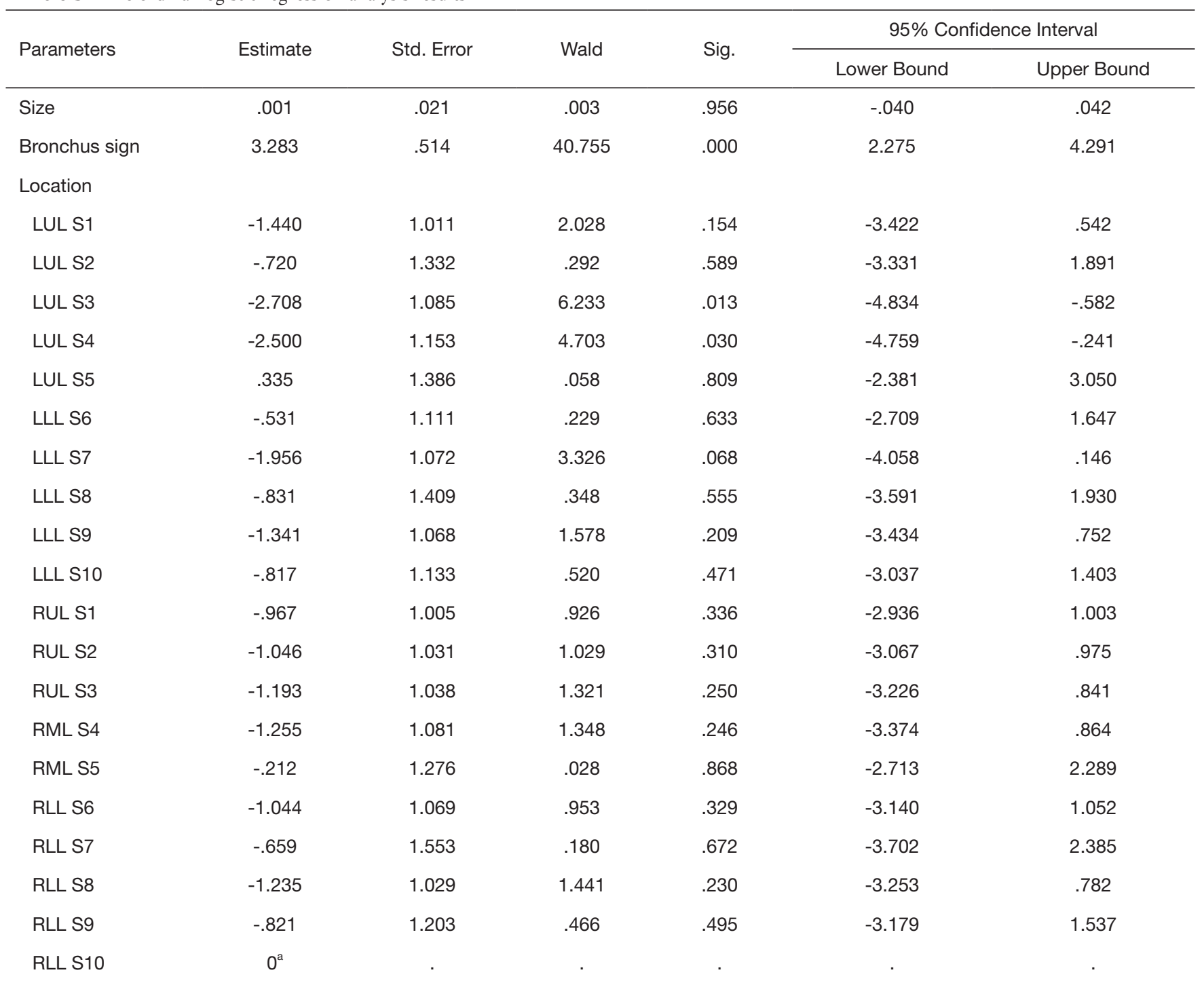

${ }^{a}$ This parameter is set to zero because it is redundant. RUL, right upper lobe; RML, right middle lobe; RLL, right lower lobe; LUL, left upper lobe; LLL, left lower lobe. 